\title{
THREE-DIMENSIONAL OSEEN FLOW PAST A FLAT PLATE*
}

\author{
BY
}

\author{
DOREL HOMENTCOVSCHI
}

Central Institute of Mathematics, Bucharest

\begin{abstract}
On the basis of the Oseen approximation the three-dimensional flow of a viscous incompressible fluid past a flat plate is studied. A system of two integral equations for determining the drag and the lateral force on the plate and an integral equation for the lift are obtained. The paper gives the asymptotic form of the integral equation for the lift, for high Reynolds numbers. In the inviscid limit the integral equation of the lifting surface theory is obtained. Lifting line theory and slender wing theory in weak viscous flow are discussed. Viscosity corrections are given for some particular wings: elliptic wings of high aspect ratio and slender delta wings.
\end{abstract}

1. Introduction. The steady flow of a viscous incompressible fluid past a body is one of the fundamental subjects of fluid dynamics. The governing Navier-Stokes equations are nonlinear and exact solutions have been obtained only for very specific problems. In this situation many studies have been based on the linearized equations. Thus the Oseen approximation replaces the convective (nonlinear) terms in the Navier-Stokes equations by convection due to the uniform velocity at infinity. It is adequate at large distances where the flow is almost uniform; for small Reynolds numbers it is also justified in the neighborhood of the body due to the prevalence of the diffusion terms (retained exactly in linearized equations) over the convection terms.

In the case of steady flow past a flat plate placed along the $z=0$ plane and having a small incidence angle, the correct momentum balance is also obtained from Oseen's equations at the surface of the plate, for all Reynolds numbers, because the no-slip condition applies there. For these reasons Oseen's approximation may be expected to give some correct results in the case of the flat plate even at high Reynolds numbers. For the lift things are as we expected: in the limit case of an inviscid fluid and plane motion past a flat plate the Oseen approximation furnishes correct values for the lift in subsonic as well as in supersonic flow $[3,4]$. Furthermore, in the subsonic case this theory provides a justification for the Kutta-Joukowsky condition.

For the drag things are different: the value obtained by using Oseen's approximation is different from the Blasius value [5]. This has to be expected since the Oseen model does not eliminate the paradox according to which the drag is the same irrespective of the direction of the velocity at infinity [7]. Therefore in the study of fluid motion with a small incidence angle past a flat plate placed along the $z=0$ plane, the Oseen approximation supplies corrections valid only for the lift in the limit case of the high Reynolds numbers.

* Received February 2, 1981. 
In this paper we determine the lift on a three-dimensional flat plate in the limit case of vanishing viscosity. First we obtain a new representation formula for the velocity field in the Oseen approximation. The boundary condition furnishes a system of two integral equations for the derivation of the drag and lateral force on the plate and a single integral equation for the lift. The main result of the paper is the asymptotic expansion of the kernel of this integral equation, for high Reynolds numbers. The zeroth-order term in this expansion is just the kernel of the integral equation of the lifting surface theory in aerodynamics; the next term is a correction due to viscosity which is important in wing theory in aerodynamics as well as in hydrofoil hydrodynamics.

The last two sections give some applications: we have developed a "lifting line" theory and a "slender wing" theory for weak viscous flow. The paper gives corrective coefficients for the lift in these approximations for some specific wings. For very slender wings the viscosity plays an important role in determining the lift since it appears in the leading term in the asymptotic expansion.

2. Basic equations. We consider the steady motion of an incompressible viscous fluid around a three-dimensional body. The fluid motion is governed by the Navier-Stokes equations. The form of these equations has been deduced in [8]; in our case they are:

$$
\begin{gathered}
\operatorname{div} \mathbf{V}=0, \\
(\mathbf{V} \operatorname{grad}) \mathbf{V}+\frac{1}{\rho} \operatorname{grad} P-v \Delta \mathbf{V}=\frac{1}{\rho} \mathbf{T} \delta_{s} .
\end{gathered}
$$

Here $\mathbf{V}\left(V_{x}, V_{y}, V_{z}\right)$ is the fluid velocity, $P$ is the pressure, $\rho$ the density, and $v$ the kinematic viscosity. The term $\mathbf{T} \delta_{S}$ describes the body action on the fluid motion. Consequently the resultant $\mathbf{R}$ and the resultant momentum $\mathbf{M}$ of the forces acting upon the body may be expressed, taking into account the right-hand side of $(2.1)$, by the relations

$$
\begin{gathered}
\mathbf{R}=\left\langle-\mathbf{T} \delta_{S}, 1\right\rangle=-\iint_{S} \mathbf{T} d \sigma, \\
\mathbf{M}=\left\langle-\mathbf{x} \times \mathbf{T} \delta_{S}, 1\right\rangle=-\iint_{S} \mathbf{x} \times \mathbf{T} d \sigma .
\end{gathered}
$$

We denote by $L$ the characteristic length of the body and by $\mathbf{V}_{0}$ the velocity of the uniform flow at infinity upstream. The spatial coordinates $x_{1}, y_{1}, z_{1}$ are taken so that $\mathbf{V}_{0}$ is in the $x_{1} 0_{1} z_{1}$ plane and makes a small angle $\theta_{0}$ with the $0 x_{1}$ axes. Denoting by $\mathbf{V}^{\prime}, p^{\prime}$ the velocity and pressure perturbations respectively, we introduce the following dimensionless quantities:

$$
x=\frac{x_{1}}{L}, \quad y=\frac{y_{1}}{L}, \quad z=\frac{z_{1}}{L}, \quad \mathbf{v}=\frac{\mathbf{V}^{\prime}}{V_{0}}, \quad p=\frac{p^{\prime}}{\rho V_{0}^{2}}, \quad \mathbf{t}=\frac{\mathbf{T}}{\rho V_{0}^{2}} .
$$

By assuming small perturbations we shall linearize Eq. (2.2) about the undisturbed state by neglecting the product of perturbations and the product of perturbations by $\sin \theta_{0}$. The resulting equations are similar in form to Oseen's approximation:

$$
\begin{gathered}
\operatorname{div} \mathbf{v}=0 \\
\frac{\partial \mathbf{v}}{\partial x}+\operatorname{grad} p-\frac{1}{4 \sigma} \Delta \mathbf{v}=\mathbf{t} \delta_{S},
\end{gathered}
$$


where

$$
4 \sigma=\frac{L \rho V_{0}}{\mu}
$$

is the Reynolds number.

At infinity all the perturbations must vanish:

$$
\lim _{x^{2}+y^{2}+z^{2} \rightarrow \infty}(v, p)=0
$$

and we consider the no-slip condition on the body

$$
\begin{gathered}
v_{x}(x, y, z)=-1 \\
v_{y}(x, y, z)=0 \quad(x, y, z) \in S \\
v_{z}(x, y, z)=-\theta_{0} .
\end{gathered}
$$

We now take the Fourier transform of the system (2.6), (2.7) with respect to all spatial variables. The result is the system

$$
\begin{gathered}
i \mathbf{k} \cdot \tilde{\mathbf{v}}=0, \\
\left(i k_{1}+\frac{k^{2}}{4 \sigma}\right) \tilde{\mathbf{v}}+i \mathbf{k} \tilde{p}=\mathscr{F}\left[\mathbf{t} \delta_{s}\right],
\end{gathered}
$$

where

$$
\tilde{\mathbf{v}} \equiv \mathscr{F}[\mathbf{v}]=\iiint \mathbf{v}(x, y, z) \exp \left\{-i\left(k_{1} x+k_{2} y+k_{3} z\right)\right\} d x d y d z
$$

and similarly $\tilde{p}=\mathscr{F}[p(x, y, z)]$. The solution of the linear system $(2.13),(2.14)$ is

$$
\begin{gathered}
\tilde{p}=\frac{i \mathbf{k}}{k^{2}} \mathscr{F}\left[\mathbf{t} \delta_{s}\right], \\
\tilde{\mathbf{v}}=\frac{1}{i k_{1}}\left\{\frac{1}{k^{2}}-\frac{1}{k^{2}+4 \sigma i k_{1}}\right\}\left\{i \mathbf{k}\left(i \mathbf{k} \cdot \mathscr{F}\left[\mathbf{t} \delta_{s}\right]\right)+k^{2} \mathscr{F}\left[\mathbf{t} \delta_{s}\right]\right\} .
\end{gathered}
$$

To determine the inverse transform of the solution we use the relations

$$
\begin{gathered}
\mathscr{F}^{-1}\left[\frac{1}{k^{2}+4 \sigma i k_{1}}\right]=\frac{\exp \{2 \sigma(x-|\mathbf{x}|)\}}{4 \pi|\mathbf{x}|}, \\
\int_{-\infty}^{x} \frac{\partial}{\partial y} \frac{\exp \{2 \sigma(x-|\mathbf{x}|)\}}{4 \pi|\mathbf{x}|} d x=-\frac{1}{4 \pi} \frac{y}{y^{2}+z^{2}}\left(1+\frac{\mathbf{x}}{|\mathbf{x}|}\right) \exp \{2 \sigma(x-|\mathbf{x}|)\} .
\end{gathered}
$$

Finally we obtain

$$
\begin{gathered}
p(x, y, z)=\frac{\partial}{\partial x} \frac{1}{4 \pi|\mathbf{x}|} \circledast f \delta_{S}+\frac{\partial}{\partial y} \frac{1}{4 \pi|\mathbf{x}|} \circledast g \delta_{s}+\frac{\partial}{\partial z} \frac{1}{4 \pi|\mathbf{x}|} \circledast h \delta_{s}, \\
v_{x}(x, y, z)=4 \sigma \frac{\exp \{2 \sigma(x-|\mathbf{x}|)\}}{4 \pi|\mathbf{x}|} \circledast f \delta_{s}+\frac{\partial}{\partial x} \frac{1-\exp \{2 \sigma(x-|\mathbf{x}|)\}}{4 \pi|\mathbf{x}|} \circledast f \delta_{S} \\
+\frac{\partial}{\partial y} \frac{1-\exp \{2 \sigma(x-|\mathbf{x}|)\}}{4 \pi|\mathbf{x}|} \circledast g \delta_{s}+\frac{\partial}{\partial z} \frac{1-\exp \{2 \sigma(x-|\mathbf{x}|)\}}{4 \pi|\mathbf{x}|} \circledast h \delta_{s},
\end{gathered}
$$




$$
\begin{aligned}
v_{y}(x, y, z)=\frac{\partial}{\partial y} & \frac{1-\exp \{2 \sigma(x-|\mathbf{x}|)\}}{4 \pi|\mathbf{x}|} \circledast f \delta_{S}+4 \sigma \frac{\exp \{2 \sigma(x-|\mathbf{x}|)\}}{4 \pi|\mathbf{x}|} \circledast g \delta_{S} \\
& -\frac{1}{4 \pi} \frac{\partial}{\partial y}\left\{\frac{y}{y^{2}+z^{2}}\left(1+\frac{x}{|\mathbf{x}|}\right)(1-\exp \{2 \sigma(x-|\mathbf{x}|)\})\right\} \circledast g \delta_{S} \\
& -\frac{1}{4 \pi} \frac{\partial}{\partial y}\left\{\frac{z}{y^{2}+z^{2}}\left(1+\frac{x}{|\mathbf{x}|}\right)(1-\exp \{2 \sigma(x-|\mathbf{x}|)\})\right\} \circledast h \delta_{S}, \\
v_{z}(x, y, z)= & \frac{\partial}{\partial z} \frac{1-\exp \{2 \sigma(x-|\mathbf{x}|)\}}{4 \pi|\mathbf{x}|} \circledast f \delta_{S} \\
& -\frac{1}{4 \pi} \frac{\partial}{\partial z}\left\{\frac{y}{y^{2}+z^{2}}\left(1+\frac{x}{|\mathbf{x}|}\right)(1-\exp \{2 \sigma(x-|\mathbf{x}|)\})\right\} \circledast g \delta_{S} \\
& -\frac{1}{4 \pi} \frac{\partial}{\partial z}\left\{\frac{z}{y^{2}+z^{2}}\left(1+\frac{x}{|\mathbf{x}|}\right)(1-\exp \{2 \sigma(x-|\mathbf{x}|)\})\right\} \circledast h \delta_{S} \\
& +4 \sigma \frac{\exp \{2 \sigma(x-|\mathbf{x}|)\}}{4 \pi|\mathbf{x}|} \circledast h \delta_{S} .
\end{aligned}
$$

We denote by $\circledast$ the convolution product with respect to all the spatial variables; $\mathbf{t}=f \mathbf{i}+g \mathbf{j}+h \mathbf{k}$.

Formulae (2.19) $-(2.22)$ are a representation for the solution of the Oseen system in the case of an arbitrary body; the unknown densities $f, g, h$ are just the quantities of interest in most hydrodinamic problems. In order to determine these functions the boundary conditions (2.10)-(2.12) will be used. They will furnish a system of three integral equations for solving the problem.

3. Integral equations for the motion past a flat plate. Let us now study the motion of the viscous fluid past a flat plate. We take the axes such that the $0 x y$ plane coincides with the plane of the plate and the velocity at infinity is in the 0xz plane. By taking $z \rightarrow 0$ in relations $(2.20)-(2.22)$ we get

$$
\begin{aligned}
& v_{x}(x, y, 0)=\left\{-\frac{x}{4 \pi r^{3}}(1-\exp \{2 \sigma(x-r)\})+\frac{\sigma}{\pi r}\left(1+\frac{x}{r}\right) \exp \{2 \sigma(x-r)\}\right\} * f(x, y) \\
& +\left\{\frac{\sigma y}{2 \pi r^{2}} \exp \{2 \sigma(x-r)\}-\frac{y}{4 \pi r^{3}}(1-\exp \{2 \sigma(x-r)\})\right\} * g(x, y), \\
& v_{y}(x, y, 0)=\left\{-\frac{y}{4 \pi r^{3}}(1-\exp \{2 \sigma(x-r)\})+\frac{\sigma y}{2 \pi r^{2}} \exp \{2 \sigma(x-r)\}\right\} * f(x, y) \\
& +\left\{\frac{1}{4 \pi y^{2}}\left(1+\frac{x}{r}\right)(1-\exp \{2 \sigma(x-r)\})+\frac{x}{4 \pi r^{3}}(1-\exp \{2 \sigma(x-r)\})\right. \\
& \left.+\frac{\sigma}{2 \pi r}\left(1-\frac{x}{r}\right) \exp \{2 \sigma(x-r)\}\right\} * g(x, y),
\end{aligned}
$$

$v_{z}(x, y, 0)=\left\{-\frac{1}{4 \pi y^{2}}\left(1+\frac{x}{r}\right)(1-\exp \{2 \sigma(x-r)\})+\frac{\sigma}{\pi r} \exp \{2 \sigma(x-r)\}\right\} * h(x, y)$,

where $*$ is now the convolution product with respect to $x$ and $y$ and $r=\left(x^{2}+y^{2}\right)^{1 / 2}$. The 
unknown densities are nonvanishing only on the plate; hence all the convolution products above exist.

Taking into account the relations (3.1)-(3.3) the boundary conditions become:

$$
\begin{aligned}
\frac{1}{4 \pi} \iint_{S} f(\xi, \eta)\left\{\frac{2 X}{R^{3}}\right. & \left.\exp \{\sigma(X-R)\} \sinh \{\sigma(X-R)\}+\frac{2 \sigma}{R}\left(1+\frac{X}{R}\right) \exp \{2 \sigma(X-R)\}\right\} d \xi d \eta \\
& +\frac{1}{4 \pi} \iint_{S} g(\xi, \eta)\left\{\frac{2 Y}{R^{3}} \exp \{\sigma(X-R)\} \sinh \{\sigma(X-R)\}\right. \\
& \left.+\frac{2 \sigma Y}{R^{2}} \exp \{2 \sigma(X-R)\}\right\} d \xi d \eta=-1, \quad(x, y) \in S,
\end{aligned}
$$$$
\frac{1}{4 \pi} \iint_{S} f(\xi, \eta)\left\{\frac{2 Y}{R^{3}} \exp \{\sigma(X-R)\} \sinh \{\sigma(X-R)\}+\frac{2 \sigma Y}{R^{2}} \exp \{2 \sigma(X-R)\}\right\} d \xi d \eta
$$$$
+\frac{1}{4 \pi} \iint_{S} g(\xi, \eta)\left\{-\frac{2 X}{R^{3}} \exp \{\sigma(X-R)\} \sinh \{\sigma(X-R)\}+\frac{2 \sigma}{R}\left(1-\frac{X}{R}\right) \exp \{2 \sigma(X-R)\}\right.
$$$$
\left.+\frac{2 \sigma}{R} \exp \{\sigma(X-R)\} \frac{\sinh \{\sigma(X-R)\}}{(X-R)}\right\} d \xi d \eta=0, \quad(x, y) \in S,
$$

$$
\begin{aligned}
\frac{1}{4 \pi} \iint_{S} h(\xi, \eta)\left\{\frac{4 \sigma}{R} \exp \{2 \sigma(X-R)\}\right. & -\frac{2 \sigma}{R} \exp \{\sigma(X-R)\} \\
& \left.\cdot \frac{\sinh \{\sigma(X-R)\}}{\sigma(X-R)}\right\} d \xi d \eta=-\theta_{0}, \quad(x, y) \in S .
\end{aligned}
$$

where $X=x-\xi, Y=y-\eta, R=\left(X^{2}+Y^{2}\right)^{1 / 2}$.

The relations (3.4), (3.5) are a system of integral equations for the densities $f(x, y)$ and $g(x, y)$. The integral equation (3.6) determines the local lift $h(x, y)$.

The kernels of all integral equations obtained have weak singularities. However, closed analytical solutions cannot be obtained such that these equations must be solved numerically or asymptotically.

4. Asymptotic form of Eq. (3.6) for high Reynolds numbers. In order to obtain the asymptotic form of Eq. (3.6) we shall consider its kernel as a distribution in $\mathscr{D}^{\prime}\left(R^{2}\right)$ depending upon the parameter $\sigma[1]$. Let

$$
I(\sigma)=\left\langle\frac{4 \sigma \exp \{2 \sigma(x-r)\}}{r}-\frac{\exp \{2 \sigma(x-r)\}-1}{r(x-r)}, \varphi(x, y)\right\rangle, \quad \varphi \in \mathscr{D}\left(R^{2}\right)
$$

We have

$$
\begin{aligned}
I(\sigma) & =\iint\left(\frac{4 \sigma \exp \{2 \sigma(\dot{x}-r)\}}{r}-\frac{\exp \{2 \sigma(x-r)\}-1}{r(x-r)}\right) \varphi(x, y) d x d y \\
& =\int_{0}^{2 \pi} \int_{0}^{\infty}(4 \sigma \exp \{-2 \sigma r(1-\cos \theta)\}
\end{aligned}
$$




$$
\left.+\frac{\exp \{-2 \sigma r(1-\cos \theta)\}-1}{r(1-\cos \theta)}\right) \varphi(r \cos \theta, r \sin \theta) d r d \theta .
$$

Let $\phi(\theta)$ be an "unitary function" [6], i.e. a function of $\mathscr{D}(R)$ such that $\sum_{n=-\infty}^{\infty} \phi(\theta$ $-2 n \pi)=1$. We can write

$$
\begin{aligned}
I(\sigma)= & \int_{-\infty}^{\infty} \int_{0}^{\infty}\left(4 \sigma \exp \{-2 \sigma r(1-\cos \theta)\}+\frac{\exp \{-2 \sigma r(1-\cos \theta)\}-1}{r(1-\cos \theta)}\right) \\
& \cdot \varphi(r \cos \theta, r \sin \theta) \phi(\theta) d r d \theta \\
= & \int_{0}^{\infty} 4 \sigma \exp \left\{-2 \sigma c^{2}\right\} d c \int_{G=c} \phi(\theta) \varphi(r \cos \theta, r \sin \theta) \omega \\
& +\int_{0}^{\infty} \frac{\exp \left\{-2 \sigma c^{2}\right\}-1}{c^{2}} d c \int_{G=c} \phi(\theta) \varphi(r \cos \theta, r \sin \theta) \omega
\end{aligned}
$$

where $G(r, \theta)=(r(1-\cos \theta))^{1 / 2}$ and $\omega$ is the differential form on the manifold $G=c$ defined by the equation $d G \omega=d r d \theta$.

Let us define

$$
J(c)=\int_{G=c} \phi(\theta) \varphi(r \cos \theta, r \sin \theta) \omega .
$$

In order to obtain the asymptotic expansion of the function $I(\sigma)$ we need the asymptotic expansion of the function $J(c)$ for small values of $c$. To get this expansion we first consider the function

$$
G(\lambda)=\left\langle G_{+}^{\lambda}, \psi(r, \theta)\right\rangle=\iint_{G^{2}>0}[r(1-\cos \theta)]^{\lambda / 2} \psi(r, \theta) d r d \theta, \quad \psi(r, \theta) \in \mathscr{D}\left(R^{2}\right) .
$$

It is an analytic function of $\lambda$, holomorphic for $\operatorname{Re}\{\lambda\}>0$ and its singularities are intimately related to the nature of the manifold $G=0$.

Let $M$ be a point on the submanifold $r=0$ and $\psi(r, \theta)=0$ outside some fixed small neighborhood of $M$. We have

$$
G(\lambda)=\int_{0}^{\infty} \xi^{\lambda / 2} d \xi \int_{-\infty}^{+\infty} \psi\left(\frac{\xi}{1-\cos \theta}, \theta\right) \frac{d \theta}{1-\cos \theta} .
$$

Hence (4.6) has simple poles at $\lambda / 2=-1,-2,-3, \ldots$ and the residue of $G(\lambda)$ at $\lambda=-2 k$ $(k=1,2,3, \ldots)$ is

$$
\text { Residue }\{G(\lambda),-2 k\}=\frac{1}{(k-1) !}\left\{\frac{d^{k-1}}{d \xi^{k-1}} \int_{-\infty}^{+\infty} \psi\left(\frac{\xi}{1-\cos \theta}, \theta\right) \frac{d \theta}{1-\cos \theta}\right\}_{\xi=0} .
$$

If now $M$ is a point on the submanifold $\theta=0$ and $\psi(r, \theta)$ a function in $\mathscr{D}\left(R^{2}\right)$ with support in an arbitrarily small neighborhood of this point, we can write:

$$
G(\lambda)=\int_{-\infty}^{+\infty}|\xi|^{\lambda} d \xi \int_{0}^{\infty} \psi\left(r, \arccos \frac{r-\xi^{2}}{r}\right) \frac{2 d r}{\left(2 r-\xi^{2}\right)^{1 / 2}},
$$

and consequently the function $G(\lambda)$ has simple poles at the points $\lambda=-1,-3,-5, \ldots$ We also have 


$$
\text { Residue }\{G(\lambda),-2 k-1\}=\frac{2}{(2 k) !}\left\{\frac{d^{2 k}}{d \xi^{2 k}} \int_{0}^{\infty} \psi\left(r, \arccos \frac{r-\xi^{2}}{r}\right) \frac{2 d r}{\left(2 r-\xi^{2}\right)^{1 / 2}}\right\}_{\xi=0} .
$$

Finally, in a neighborhood of the point $M(0,0)$ we have

$$
G(\lambda)=\int_{0}^{\infty} \int_{-\infty}^{+\infty} \xi_{1}^{\lambda / 2} \xi_{2}^{\lambda} \psi\left(\xi_{1}, \arccos \left(1-\xi_{2}^{2}\right)\right) \frac{2 d \xi_{1} d \xi_{2}}{\left(2-\xi_{2}^{2}\right)^{1 / 2}}
$$

and in polar coordinates $\xi_{2}=\rho \cos \eta, \xi_{1}=\rho \sin \eta$

$$
G(\lambda)=\int_{0}^{\infty} \rho^{(3 \lambda+2) / 2} d \rho \int_{0}^{\pi}(\sin \eta)^{\lambda / 2}|\cos \eta|^{\lambda} \frac{2 \psi\left(\rho \sin \eta, \arccos \left(1-\rho^{2} \cos ^{2} \eta\right)\right)}{\sqrt{2-\rho^{2} \cos ^{2} \eta}} d \eta .
$$

Hence (4.11) has simple poles at the points $\lambda_{k}=-(2 k+2) / 3(k=1,2,3, \ldots)$. Its residue at the pole $\lambda_{k}$ is

$$
\text { Residue }\left\{G(\lambda),-\frac{2 k+2}{3}\right\}=\frac{1}{k !} \sum_{\alpha+\beta=k} \frac{\partial^{k} \tilde{\psi}(0,0)}{\partial \xi_{1}^{\alpha} \partial \xi_{2}^{\beta}} \int_{\Gamma}^{*} \frac{\xi_{1}^{\alpha} \xi_{2}^{\beta}\left(\xi_{1} d \xi_{2}-\xi_{2} d \xi_{1}\right)}{\xi_{1}^{(k+1) / 3} \xi_{2}^{2(k+1) / 3}} .
$$

Here $\Gamma$ is the upper semicircle $\rho=1$, the integral $\int^{*}$ is the regularization of the corresponding integral and $\tilde{\psi}(\rho, \eta)=\psi\left(\rho \sin \eta, \arccos \left(1-\rho^{2} \cos ^{2} \eta\right)\right)$.

The above analysis shows that the function $G(\lambda)$ defined by (4.5) for $\operatorname{Re}\{\lambda\}>0$ can be continued to the entire plane $\lambda$ as a meromorphic function with poles at the points of the above sequences; at the common points to the first and third sequences (i.e. $\lambda=-2 k$, $k=1,2,3, \ldots)$ it has double poles and at the remaining singular points it has simple poles.

We consider now the function

$$
H(\lambda)=\left\langle G_{+}^{\lambda}, \phi(\theta) \varphi(r \cos \theta, \sin \theta)\right\rangle .
$$

We have

$$
\begin{gathered}
\text { Residue }\{H(\lambda),-2\}=\varphi(0,0) \int_{0}^{2 \pi_{*}} \frac{d \theta}{1-\cos \theta}=0 \\
\text { Residue }\left\{H(\lambda),-\frac{2 k+2}{3}\right\}=0 \\
\text { Residue }\{H(\lambda),-1\}=2 \sqrt{ } 2 \int_{0}^{\infty} \frac{\varphi(r, 0)}{\sqrt{r}} d r \equiv a_{1} \\
\operatorname{Residue}\{H(\lambda),-3\}=\int_{0}^{\infty *}\left(2 \sqrt{2 r} \frac{\partial^{2} \varphi(r, 0)}{\partial y^{2}}-\frac{4}{\sqrt{2 r}} \frac{\partial \varphi(r, 0)}{\partial r}+\frac{2 \varphi(r, 0)}{(\sqrt{2 r})^{3}}\right) d r \equiv a_{2} .
\end{gathered}
$$

The function $H(\lambda)$ can be continued to a meromorphic function in all the complex plane $\lambda$. It has only simple poles at the points $\lambda=-1,-3,-5, \ldots$. Hence we can write

$$
H(\lambda)=\frac{a_{1}}{\lambda+1}+\frac{a_{2}}{\lambda+3}+\frac{a_{3}}{\lambda+5}+\tilde{H}(\lambda)
$$

where $\tilde{H}(\lambda)$ is a holomorphic function in the domain $\operatorname{Re}\{\lambda\}>-7$ and, at the same time,

$$
\lim _{\left|\lambda_{2}\right| \rightarrow \infty} H\left(\lambda_{1}+i \lambda_{2}\right)=0 \quad \text { for } \quad-7<\lambda_{1}<0 .
$$


We can also write

$$
\begin{gathered}
a_{1}=\left\langle\frac{2 \sqrt{ } 2}{\sqrt{ } x} \theta_{+}(x) \delta(y), \varphi(x, y)\right\rangle, \\
a_{2}=\left\langle 2 \sqrt{ } 2 x \theta_{+}(x) \delta^{\prime \prime}(y)-\frac{\sqrt{ } 2 \theta_{+}(x)}{2 x \sqrt{x}} \delta(y), \varphi(x, y)\right\rangle,
\end{gathered}
$$

$\theta_{+}(x)$ being the Heaviside function. We have

$$
H(\lambda)=\int_{0}^{\infty} c^{\lambda} d c \int_{G=c}\left(\varphi(\theta) \varphi(r \cos \theta, r \sin \theta) \omega=\int_{0}^{\infty} c^{\lambda} J(c) d c\right.
$$

Hence the function $H(\lambda)$ is the Mellin transform of the function $c J(c)$. We use the inversion formula

$$
J(c)=\frac{1}{2 \pi i} \int_{\sigma-i \infty}^{\sigma+i \infty} c^{-\lambda-1} H(\lambda) d \lambda, \quad \sigma>0 .
$$

Let $-7<-b<-5$. The residue theorem gives

$$
J(c)=\sum_{k=0}^{2} \operatorname{Residue}\left\{c^{-\lambda-1} H(\lambda),-(2 k+1)\right\}+\frac{1}{2 \pi i} \int_{-b-i \infty}^{-b+i \infty} c^{-\lambda-1} H(\lambda) d \lambda .
$$

The last integral in this relation has the order $O\left(c^{b-\varepsilon}\right)$, with $\varepsilon$ arbitrarily small. The relation (4.24) gives the asymptotic expansion of the function $J(c)$ :

$$
J(c)=a_{1}+a_{2} c^{2}+O\left(c^{4}\right) .
$$

The asymptotic expansion of the first term in (4.3) can be obtained now by using Watson's Lemma :

$$
I_{1} \equiv \int_{0}^{\infty} 4 \sigma \exp \left\{-2 \sigma c^{2}\right\} J(c) d c=\sqrt{2 \pi \sigma} a_{1}+\frac{\sqrt{ } \pi}{2 \sqrt{2 \sigma}} a_{2}+O\left(\sigma^{-3 / 2}\right)
$$

For the second term we write

$$
\begin{aligned}
I_{2} & \equiv \int_{0}^{\infty} \frac{\exp \left\{-2 \sigma c^{2}\right\}-1}{c^{2}} J(c) d c \\
& =\int_{0}^{\infty} \frac{\exp \left\{-2 \sigma c^{2}\right\}-1}{c^{2}}\left(J(c)-a_{1}\right) d c+a_{1} \int_{0}^{\infty} \frac{\exp \left\{-2 \sigma c^{2}\right\}-1}{c^{2}} d c .
\end{aligned}
$$

Hence

$$
\begin{aligned}
I_{2} & =\int_{0}^{\infty} \frac{J(c)-a_{1}}{c^{2}} \exp \left\{-2 \sigma c^{2}\right\} d c-\int_{0}^{\infty} \frac{J(c)-a_{1}}{c^{2}} d c-a_{1} \sqrt{2 \pi \sigma} \\
& =-\sqrt{2 \pi \sigma} a_{1}+\frac{\sqrt{\pi}}{2 \sqrt{2 \sigma}} a_{2}-\int_{0}^{\infty} \frac{J(c)}{c^{2}} d c+O\left(\sigma^{-3 / 2}\right)
\end{aligned}
$$


Finally the kernel of Eq. (3.6) has the asymptotic expansion

$$
\begin{aligned}
\frac{4 \sigma \exp \{2 \sigma(x-r)\}}{r} & -\frac{\exp \{2 \sigma(x-r)\}-1}{r(x-r)} \\
& =-\frac{1}{y^{2}}\left(1+\frac{x}{r}\right)+\frac{\sqrt{ } \pi}{\sqrt{ } \sigma}\left(2 \sqrt{ } x \theta_{+}(x) \delta^{\prime \prime}(y)-\frac{\theta_{+}(x)}{2 x \sqrt{ } x} \delta(y)\right)+O\left(\sigma^{-3 / 2}\right) .
\end{aligned}
$$

Consequently Eq. (3.6) gives

$$
\begin{aligned}
& -\frac{1}{4 \pi} \int_{S}^{*} \int_{S} \frac{h(\xi, \eta)}{(y-\eta)^{2}}\left(1+\frac{x-\xi}{\sqrt{(x-\xi)^{2}+(y-\eta)^{2}}}\right) d \xi d \eta \\
& \quad+\frac{1}{4 \sqrt{\pi \sigma}}\left\{\int_{x_{-}}^{x} 2 \sqrt{x-\xi} \frac{\partial^{2} h(\xi, y)}{\partial y^{2}} d \xi-\int_{x_{-}}^{x_{*}} \frac{h(\xi, y) d \xi}{2(x-\xi)^{3 / 2}}\right\}+O\left(\sigma^{-3 / 2}\right)=-\theta_{0} .
\end{aligned}
$$

The first term is well known in the theory of the lifting surface and the second one is a correction due to viscosity for high Reynolds numbers. If in (4.30) the spatial variables are replaced by dimensional ones then $\sigma^{\prime}=\rho \mu^{-1} V_{0}$ stands for $\sigma$.

5. Lifting line theory in the flow of a weak viscous fluid. Let now the surface $S$ be a plane wing of large aspect ratio. We denote by $2 a$ the chord and by $2 b$ the span of the wing and perform the changes of variables

$$
\begin{array}{ll}
x=a x^{\prime}, & \xi=a \xi^{\prime} ; \\
y=b y^{\prime}, & \eta=b \eta^{\prime} .
\end{array}
$$

Again we denote by $x, y, \xi, \eta$ the independent variables, by $S$ the domain resulting from $S$ by the transformation $(5.1)$ and by $h(x, y)$ the new unknown function. Eq. (4.30) becomes:

$$
\begin{gathered}
\frac{\varepsilon}{4 \pi} \iint_{S} \frac{\partial h(\xi, \eta)}{\partial \eta} \frac{d \xi d \eta}{y-\eta}+\frac{1}{4 \pi} \iint_{S} \frac{\partial h(\xi, \eta)}{\partial \eta} \frac{\sqrt{\varepsilon^{2}(x-\xi)^{2}+(y-\eta)^{2}}}{(x-\xi)(y-\eta)} d \xi d \eta \\
+\frac{1}{4 \sqrt{\pi \sigma^{\prime} a}}\left\{\varepsilon^{2} \int_{x_{-}}^{x} 2 \sqrt{x-\xi} \frac{\partial^{2} h(\xi, y)}{\partial y^{2}} d \xi-\int_{x_{-}}^{x} \frac{h(\xi, y)}{2\left(\sqrt{x-\xi)^{3}}\right.} d \xi\right\} \\
+O\left(\sigma^{-3 / 2}\right)=-\theta_{0}, \quad(x, y) \in S,
\end{gathered}
$$

where $\varepsilon=a / b$. We consider now the asymptotic form for $\varepsilon \ll 1$, i.e. for large-aspect-ratio wings. The first terms of the asymptotic expansion of the second integral in (5.2) are given in [2]. We obtain

$$
\begin{aligned}
\frac{1}{4 \pi} \int_{x_{-}(y)}^{x+(y)} \frac{h(\xi, y)}{x-\xi} d \xi & -\frac{\varepsilon}{4 \pi} \iint_{S} \frac{h(\xi, \eta)}{(y-\eta)^{2}} d \eta \\
& -\frac{1}{8 \sqrt{\pi \sigma^{\prime} a}} \int_{x-(y)}^{x} \frac{h(\xi, y)}{(\sqrt{x-\xi})^{3}} d \xi+O\left(\sigma^{-3 / 2}\right)+O\left(\varepsilon^{2} \ln \varepsilon\right)=-\theta_{0} .
\end{aligned}
$$


For $\sigma^{\prime} \rightarrow \infty$ Eq. (5.3) coincides with the lifting line equation and for $\varepsilon \rightarrow 0$ we obtain the equation of plane motion in a weak viscous flow [3]. We have

$$
\begin{aligned}
& h(x, y)-\frac{\varepsilon}{2 \pi} \sqrt{\frac{x_{+}(y)-x}{x-x_{-}(y)}} \int_{-1}^{1} \frac{h(\eta)}{(y-\eta)^{2}} d \eta=-2 \theta_{0} \sqrt{\frac{x_{+}(y)-x}{x-x_{-}(y)}} \\
& +\frac{1}{4 \pi \sqrt{\pi \sigma^{\prime} a}} \sqrt{\frac{x_{+}(y)-x}{x-x_{-}(y)}} \int_{x_{-}(y)}^{x+(y)} \sqrt{\frac{t-x_{-}(y)}{x_{+}(y)-t}}\left\{\int_{x_{-}(y)}^{t} \frac{h(\xi, y) d \xi}{(\sqrt{t-\xi})^{3}}\right\} \frac{d t}{t-x} \\
& h(y)=\int_{x_{-}}^{x_{+}} h(\xi, y) d \xi
\end{aligned}
$$

and $x_{ \pm}=x_{ \pm}(y)$. By integration with respect to $x$ the integral equation (5.4) gives

$$
\begin{aligned}
h(y)-\varepsilon \frac{x_{+}-x_{-}}{2} \int_{-1}^{1} \frac{h(\eta)}{(y-\eta)^{2}} d \eta= & -\pi \theta_{0}\left(x_{+}-x_{-}\right) \\
& +\frac{1}{4 \sqrt{\pi \sigma^{\prime} a}} \int_{x_{-}}^{x_{+}} \sqrt{\frac{t-x_{-}}{x_{+}-t}} d t \int_{x_{-}}^{t} \frac{h(\xi, y)}{(\sqrt{t-\xi})^{3}} d \xi .
\end{aligned}
$$

Eqs. (5.4), (5.6) will be solved by the method of successive approximations. We consider $\sigma^{\prime} \rightarrow \infty$ and determine the function $h(y)$ as the solution of Eq. (5.6). It is just the solution for the classical lifting line theory. By means of the function $h(y)$ Eq. (5.4) with $\sigma^{\prime} \rightarrow \infty$ furnishes the zero term of the asymptotic expansion of the solution. By using this solution in Eqs. (5.6), (5.4) we determine the first-order term, and so on.

As an application let us consider the elliptic wing of large aspect ratio. We have

$$
x_{ \pm}(y)= \pm \sqrt{1-y^{2}} \text {. }
$$

The zero-order terms are

$$
\begin{gathered}
h_{0}(y)=-\frac{2 \theta_{0} \pi}{1+\varepsilon(\pi / 2)} \sqrt{1-y^{2}} \\
h_{0}(x, y)=-\frac{2 \theta_{0}}{1+\varepsilon(\pi / 2)}\left\{\frac{\sqrt{1-y^{2}}-x}{x+\sqrt{1-y^{2}}}\right\}^{1 / 2}
\end{gathered}
$$

Eq. (5.6) gives

$$
h_{1}(y)=-\frac{\theta_{0}}{2 \pi \sqrt{\pi \sigma^{\prime} a}} \frac{1}{1+\varepsilon(\pi / 2)} \int_{x_{-}}^{x_{+}} \sqrt{\frac{t-x_{-}}{x_{+}-t}} d t \int_{x_{-}}^{t} \sqrt{\frac{x_{+}-\xi}{\xi-x_{-}}} \frac{d \xi}{(\sqrt{t-\xi})^{3}}
$$

and hence

$$
h(y)=-\frac{2 \theta_{0}}{1+\varepsilon(\pi / 2)}\left\{\pi \sqrt{1-y^{2}}+\frac{1}{4 \pi \sqrt{\pi \sigma^{\prime} a}} k(y)\right\}
$$

where

$$
k(y)=\int_{x_{-}}^{x_{+}} \sqrt{\frac{t-x_{-}}{x_{+}-t}} d t \int_{x_{-}}^{t} \sqrt{\frac{x_{+}-\xi}{\xi-x_{-}}} \frac{d \xi}{(\sqrt{t-\xi})^{3}} .
$$




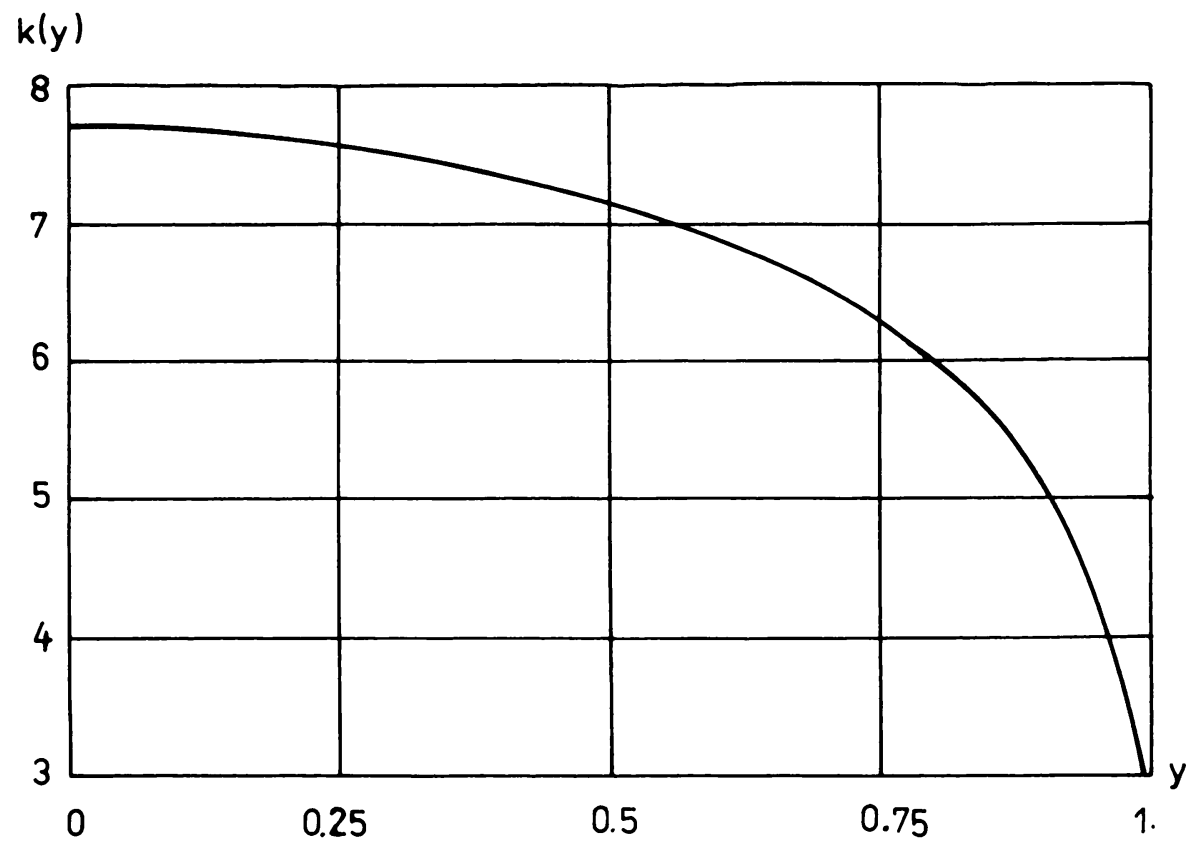

FIG. 1.

Thus the effect of the viscosity is a supplementary local lift. The function $k(y)$ is plotted in Fig. 1; note the elliptical form of the additional local lift. The overall lift is

$$
\mathscr{L}=-\rho V_{0}^{2} a b \int_{-1}^{1} h(y) d y=\frac{\rho V_{0}^{2} a b \theta_{0}}{1+\xi(\pi / 2)} \pi^{2}\left\{1+\frac{6.46}{\pi^{3} \sqrt{\pi \sigma^{\prime} a}}\right\} .
$$

6. Wings of small aspect ratio. With the notation as in the beginning of Sec. 5 we consider now the case of small aspect ratio wings, i.e., $\varepsilon \gg 1$. Eq. (5.2) gives

$$
\begin{aligned}
\frac{\varepsilon}{2 \pi} \iint_{S} \frac{\partial h(\xi, \eta)}{\partial \eta} \frac{\theta_{+}(x-\xi)}{y-\eta} d \xi d \eta+\frac{\varepsilon^{2}}{2 \sqrt{\pi \sigma^{\prime} a}} \int_{x_{-}}^{x} \sqrt{x-\xi} \frac{\partial^{2} h(\xi, y)}{\partial y^{2}} d \xi & \\
& \quad+O\left(\sigma^{-3 / 2}\right)+O\left(\varepsilon^{-2} \ln \varepsilon\right)=-\theta_{0} .
\end{aligned}
$$

To solve this integral equation we consider two limit cases when either the first or the second term on the left-hand side of Eq. (6.1) is dominant.

i) The case $\sigma^{\prime} a \gg \varepsilon^{2}$. We write Eq. (6.1) in the form

$$
\begin{aligned}
\frac{1}{\pi} \int_{y-(x)}^{y+(x)} \frac{d \eta}{y-\eta} \int_{x_{-}(\eta)}^{x} h(\xi, \eta) d \xi= & -\frac{2 \theta_{0}}{\varepsilon} y \\
& -\frac{\varepsilon}{\sqrt{\pi \sigma^{\prime} a}} \int_{x_{-(y)}}^{x} \sqrt{x-\xi} \frac{\partial h(\xi, y)}{\partial y} d \xi+c(x)
\end{aligned}
$$

We shall solve this equation by successive approximations with respect to the small parameter $\varepsilon \sqrt{\sigma^{\prime} a}$. The first term of the asymptotic expansion of the solution is just the 
solution corresponding to the theory of small-aspect-ratio wings in aerodynamics. The function $c(x)$ will be determined from the condition that the solution $h(x, y)$ have integrable singularities only; note that it does not appear in the solution. We have

$$
\int_{x_{-}(y)}^{x} h_{0}(\xi, y) d \xi=-\frac{2 \theta_{0}}{\varepsilon} \sqrt{\left(y_{+}-y\right)\left(y-y_{-}\right)}
$$

and hence

$$
h_{0}(x, y)=-\frac{2 \theta_{0}}{\varepsilon} \frac{\partial}{\partial x} \sqrt{\left(y_{+}(x)-y\right)\left(y-y_{-}(x)\right)} .
$$

In order to obtain the next term in the asymptotic expansion of the function $h(x, y)$ we replace the function $h_{0}(x, y)$ for $h(x, y)$ in the right-hand side of the relation (6.2). We get

$$
\begin{gathered}
\int_{x_{-}(y)}^{x} h_{1}(\xi, y) d \xi=\frac{2 \theta_{0}}{\pi \sqrt{\pi \sigma^{\prime} a}} \sqrt{\left(y_{+}-y\right)\left(y-y_{-}\right)} \\
\cdot \int_{y_{-}}^{y_{+}}\left\{\int_{x_{-}(\eta)}^{x} \frac{1}{4 \sqrt{x-\xi}} \frac{y_{+}(\xi)+y_{-}(\xi)-2 \eta}{\sqrt{\left(y_{+}(\xi)-\eta\right)\left(\eta-y_{-}(\xi)\right)}} d \xi\right\} \frac{1}{\sqrt{\left(y_{+}-\eta\right)\left(\eta-y_{-}\right)}} \frac{d \eta}{\eta-y} .
\end{gathered}
$$

The lift on the wing, up to the section $x=$ const., is given by the relation

$$
\begin{aligned}
L(x) & =-\rho V_{0}^{2} a b \int_{y_{-}}^{y_{+}} d y \int_{x_{-}(y)}^{x} h(x, y) d x \\
& =2 \rho V_{0}^{2} \frac{a b \theta_{0}}{\varepsilon}\left\{\frac{\pi}{8}\left(y_{+}-y_{-}\right)^{2}+\frac{\varepsilon}{\sqrt{\pi \sigma^{\prime} a}} k_{1}(x)\right\}
\end{aligned}
$$

where

$$
k_{1}(x)=\frac{1}{8} \int_{y_{-}}^{y_{+}} \frac{2 \eta-y_{-}-y_{+}}{\sqrt{\left(y_{+}-\eta\right)\left(\eta-y_{-}\right)}} d \eta \int_{x_{-}(\eta)}^{x} \frac{2 \eta-y_{+}(\xi)-y_{-}(\xi)}{\sqrt{\left(y_{+}(\xi)-\eta\right)\left(\eta-y_{-}(\xi)\right)}} \frac{d \xi}{\sqrt{x-\xi}} .
$$

We consider now the slender delta wing whose form is an isosceles triangle of small aspect ratio. We have

$$
y_{ \pm}= \pm x
$$

The formulae (6.6), (6.7) give

$$
\begin{gathered}
L(x)=\pi \rho V_{0}^{2} \frac{a b \theta_{0}}{\varepsilon} x^{2}\left\{1+\frac{\varepsilon}{\pi \sqrt{\pi \sigma^{\prime} a}} \frac{k_{1}}{\sqrt{x}}\right\}, \\
k_{1}=2 \int_{0}^{1} \frac{\tau^{2} d \tau}{\sqrt{1-\tau}} \int_{0}^{1} \frac{t^{2} d t}{\sqrt{\left(1-t^{2}\right)\left(1-\tau^{2} t^{2}\right)}} \simeq 3.7 .
\end{gathered}
$$

The second term in the brace is a correction in the lift due to viscosity for high Reynolds numbers.

ii) The case $\sigma^{\prime} a \ll \varepsilon^{2}$. Let us now write Eq. (6.1) in the form

$$
\int_{x_{-}}^{x} \sqrt{x-\xi} \frac{\partial^{2} h(\xi, y)}{\partial y^{2}} d \xi=-\frac{2 \theta_{0} \sqrt{\pi \sigma^{\prime} a}}{\varepsilon^{2}}-\frac{\sqrt{\pi \sigma^{\prime} a}}{\pi \varepsilon} \iint_{S} \frac{\partial h(\xi, \eta)}{\partial \eta} \frac{\theta_{+}(x-\xi)}{y-\eta} d \xi d \eta .
$$


We again consider the case of the slender delta wing of small aspect ratio (6.8). We have

$$
\int_{|y|}^{x} \sqrt{x-\xi} \frac{\partial^{2} h(\xi, y)}{\partial y^{2}} d \xi=-\frac{2 \theta_{0} \sqrt{\pi \sigma^{\prime} a}}{\varepsilon^{2}}-\frac{\sqrt{\pi \sigma^{\prime} a}}{\pi \varepsilon} \int_{-x}^{x} \frac{d \eta}{y-\eta} \int_{|\eta|}^{x} \frac{\partial h(\xi, \eta)}{\partial \eta} d \xi .
$$

We shall again determine the asymptotic expansion of the solution of Eq. (6.12) by successive approximation. The zeroth-order term is given by the Abel-type integral equation

$$
\int_{|y|}^{x} \sqrt{x-\xi} \frac{\partial^{2} h_{0}(\xi, y)}{\partial y^{2}} d \xi=-\frac{2 \theta_{0} \sqrt{\pi \sigma^{\prime} a}}{\varepsilon^{2}} .
$$

Its solution is

$$
\frac{\partial^{2} h_{0}(x, y)}{\partial y^{2}}=-\frac{8 \theta_{0} \sqrt{\pi \sigma^{\prime} a}}{\pi \varepsilon^{2}} \frac{\partial^{2}}{\partial x^{2}} \sqrt{x-|y|}
$$

and hence

$$
h_{0}(x, y)=-\frac{8 \theta_{0} \sqrt{\pi \sigma^{\prime} a}}{\pi \varepsilon^{2}}\left(\sqrt{x-|y|}+\frac{1}{2} \frac{|y|}{\sqrt{x}}+C_{1}(x) y+C_{2}(x)\right) .
$$

The two arbitrary functions $C_{1}(x), C_{2}(x)$ will be determined by the condition that the function $h_{0}(x, y)$ vanishes at the two lateral borders. This gives

$$
h_{0}(x, y)=-\frac{8 \theta_{0} \sqrt{\pi \sigma^{\prime} a}}{\pi \varepsilon^{2}}\left\{\sqrt{x-|y|}-\frac{1}{2 \sqrt{x}}(x-|y|)\right\} .
$$

The lift on the wing is now

$$
L=-\rho V_{0}^{2} a b \iint_{S} h(x, y) d x d y=\rho V_{0}^{2} a b \frac{8 \theta_{0} \sqrt{\pi \sigma^{\prime} a}}{3 \pi \varepsilon^{2}}+O\left(\frac{\sigma^{\prime} a}{\varepsilon^{3}}\right)
$$

The next terms are obtained by substituting $h_{0}(x, y)$ for $h(x, y)$ in the right-hand side of Eq. (6.12). Unlike the previous case, here the influence of the viscosity on the lift is dominant: now the Reynolds number enters the leading term of the asymptotic expansion in formula (6.17).

\section{REFERENCES}

[1] I. M. Gel'fand and G. E. Shilov, Generalized functions, Academic Press, New York and London, 1964

[2] D. Homentcovschi, On the deduction of Prandtl's equation, Z.A.M.M. 57, 115-116 (1977)

[3] D. Homentcovschi, Oseen flow of a compressible fluid past a flat plate, Quart. Appl. Math. 39, 221 (1981)

[4] K. Kusukawa, On the Kutta-Joukowski condition in magnetohydrodynamics, J. Phys. Soc. Japan 19, 1031-1041 (1964)

[5] J. A. Levis and G. F. Carrier, Some remarks on the flat plate boundary layer, Quart. Appl. Math. 7, 228-235 (1949)

[6] M. J. Lighthill, Introduction to Fourier analysis and generalised functions, University Press, Cambridge, 1958

[7] W. E. Olmstead and A. K. Gautesen, A new paradox in viscous hydrodynamics, Arch. Rational Mech. Anal. 29, 58-65(1968)

[8] L. Sirovich, Steady gasdynamic flows, Physics of Fluids 11, 1424-1439 (1968) 